\title{
Fingerprint Matching Based on Global Comprehensive Similarity
}

\author{
Yuliang He, Jie Tian, Senior Member, IEEE, Liang Li, Hong Chen, and Xin Yang
}

\begin{abstract}
This paper introduces a novel algorithm based on global comprehensive similarity with three steps. To describe the Euclidean space-based relative features among minutiae, we first build a minutia-simplex that contains a pair of minutiae as well as their associated textures, with its transformation-variant and invariant relative features employed for the comprehensive similarity measurement and parameter estimation, respectively. By the second step, we use the ridge-based nearest neighborhood among minutiae to represent the ridge-based relative features among minutiae. With these ridge-based relative features, minutiae are grouped according to their affinity with a ridge. The Euclidean space-based and ridge-based relative features among minutiae reinforce each other in the representation of a fingerprint. Finally, we model the relationship between transformation and the comprehensive similarity between two fingerprints in terms of histogram for initial parameter estimation. Through these steps, our experiment shows that the method mentioned above is both effective and suitable for limited memory AFIS owing to its less than $1 \mathrm{k}$ byte template size.
\end{abstract}

Index Terms-Fingerprint identification, ridge-based nearest neighborhood among minutiae, relative feature, minutia-simplex.

\section{INTRODUCTION}

A fingerprint is a pattern of ridges and valleys on the surface of a finger. It has been used for individual identification for legal purposes. Automatic fingerprint identification, which is established in modern information technology, is even applied to civilian purposes such as access control, financial security, and verification of firearm purchasers. In fact, the Automatic Fingerprint Identification Systems (AFISs) have performed well for years in controllable circumstances. However, limited fingerprint quality, nonlinear distortion, limited time, and memory expense in an offline AFIS, such as Personal Digital Assistant (PDA) and IC Card systems, are still challenging tasks in fingerprint matching.

This paper introduces an identification algorithm based on global comprehensive similarity with the view overcoming the dilemmas encountered in fingerprint matching process. The new method introduces minutiae and local ridge information in fingerprint representation. Local ridge information helps to represent a local fingerprint region and prevent matching from failing for insufficient minutiae. A minutia-simplex is built to describe a second order Euclidean space-based relative structure [1] between two minutiae and all minutia-simplexes that closely connect minutiae and ridges represent a fingerprint. So, a fingerprint is understood to be composed of many local regions, each of which is represented by one or more minutia-simplexes. If a

- Y. He, J. Tian, L. Li, and X. Yang are with the Center for Biometrics and Security Research, Key Laboratory of Complex Systems and Intelligence Science, Institute of Automation, Chinese Academy of Science, PO Box 2728, Beijing 100080, China.

E-mail: \{yuliang.he, liang.li,xin.yang\}@ia.ac.cn,tian@doctor.com.

- H. Chen is with Microsoft Research Advance Technology Center, F3 Sigma Building, ZhiChun Road No.49, Beijing, China.

E-mail: hongchen@microsoft.com.

Manuscript received 11 June 2004; revised 4 May 2005; accepted 30 Sept. 2005; published online 13 Apr. 2006.

Recommended for acceptance by A. Rangarajan.

For information on obtaining reprints of this article, please send e-mail to: tpami@computer.org, and reference IEEECS Log Number TPAMI-0297-0604. fingerprint region deforms very little, the relative features of minutiae can be aligned for matching by using rigid transformation. Two minutiae of a minutia-simplex are subject to the positional constraint. Among other relative structures, such as minutia-triplet [2], [3], it has more reliable performance in fingerprint matching because of its rich relative features. Compared with minutia-triplet [2], [3], however, minutia-simplex has a better trade-off between its performance and computational expense. It is known that Euclidean-space relative structures have their own limitation in fingerprint representation. For example, the local texture of two relative structures is different, though their relative features are similar. Therefore, ridge-based nearest neighborhood among minutiae is introduced to represent ridge-based relative features with ridge-counts between ridges and minutiae. And, minutiae are connected as a topological network with ridges. The ridge-based and Euclidean-space relative features reinforce each other in fingerprint representation. As compared with ridge-counts among minutiae in methods [2], [4], the ridge-based nearest neighborhood among minutiae is more reliably detected, although they may be influenced by outlier rejection.

In fingerprint matching, local comprehensive similarity and local transformation parameter are first obtained by coarsely matching between relative structures. Then, the relationship between the comprehensive similarity and transformation is modeled in terms of a histogram for calculating an initial transformation model. Finally, the variable bounded box method [5] is used for double-checking local comprehensive similarities. Both the histogram and variable bounded box methods globally reduce the influence of deformation on matching. For uncontrollable conditions, such as large-deformations and large-area outlier rejection, histogram and variable bounded box may be affected and, as a consequence, their parameters should be aligned accordingly.

This paper is organized as follows: In Section 2, we provide an overview of current fingerprint identification methods in literature. In Section 3, we try to manifest how our method works in fingerprint reprocessing, fingerprint 
representation, transformation model analysis, and final matching. Section 4 is an objective evaluation of the new method with the experimental results. The last section confirms the value of the proposed method and provides some prospects for the future.

\section{A Brief OVERVIEW OF FingerPRINT IDENTIFICATION METHODS}

Fingerprint identification involves a wide range of algorithms with different techniques. These schemes are based predominantly on local landmarks, exclusive global features, as well as comprehensive fingerprint features [6]. The minutiae-based matching methods, such as Jiang and Yau's [2] and Bhanu and Tan's matching and indexing methods using minutiae-triplets [3] and Gold and Rangarajan's graph matching method [4], locate minutiae and match their relative placement in an input fingerprint and a template. The minutiae-based technique is widely used because it takes less memory expense and has time saving advantages. However, a minutia set cannot characterize overall pattern of a fingerprint and a minutiae-based fingerprint matching system can hardly match two fingerprints containing a different number of unregistered minutiae. And, it is hard to further improve their performance.

The exclusive global feature-based techniques are used not only for indexing [7], [8] but also for identification [9], [10], [11], [12]. They match the global patterns of the fingerprint texture by aligning the input global features and measuring the maximum mutual global information between two fingerprints. In these methods, however, the central point should be determined with a reliable accuracy and it is difficult to deal with distortion in the fingerprints. The exclusive global information-based AFISs also need more memory to store a fingerprint template than the other two kinds of AFISs.

Comprehensive feature-based techniques [13], [14], [15] are also seen as a hybrid matching method by fusing minutiae, local features, and global features. Local features help accelerate the alignment of the unregistered minutia patterns in different sizes. Global features are used to overcome the shortage of minutiae and local features in bad quality fingerprints. With the reasonable time and memory expenses, comprehensive feature-based techniques outperform the two aforementioned kinds of matching methods. Additionally, they combine various classifiers for fingerprint matching. With the advanced hardware technology, these approaches have become popular for their good performance in acceptable memory expense in recent years.

These methods work well for the controllable environment of small deformation and small-area outlier rejection, while they are not omnipotent methods which robustly perform with some special conditions with large-deformation.

\section{Fingerprint Matching Technique Based on Global Comprehensive Similarity}

The method is a comprehensive feature-based technique with two novel aspects: First, a minutia-simplex and the ridge-based nearest neighborhood among minutiae are combined to represent the relative features among minutiae. Second, improved from our previous work [16] in

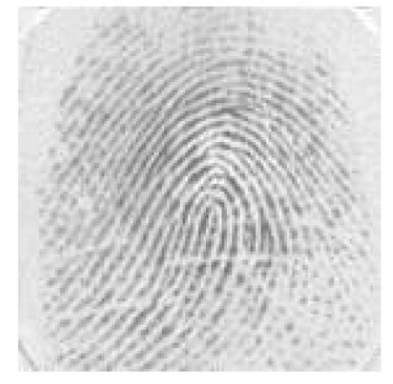

(a)

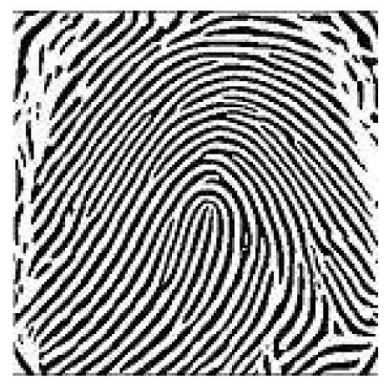

(c)

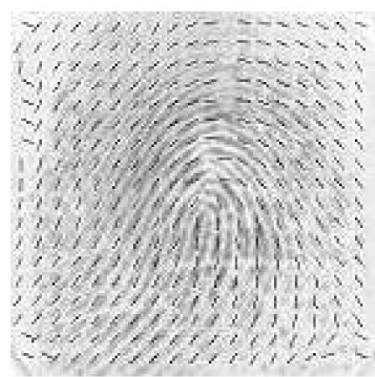

(b)

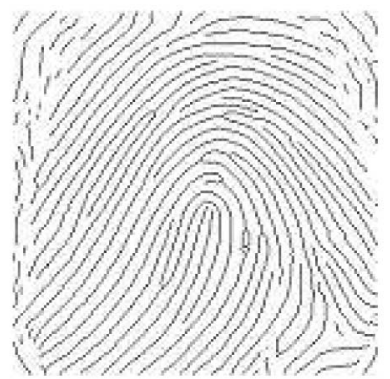

(d)
Fig. 1. Fingerprint preprocessing with our AFIS. (a) An original fingerprint in DB1_a of FVC2000, (b) its block orientation field, (c) enhanced one, and (d) thinned one.

alignment, the relationship between the comprehensive similarity and transformation is a model in terms of a histogram for estimating an initial mapping model. Compared with our previous work [16] in alignment, the new method has two novel points: Local similarity is checked with ridge-based relative features and the estimated parameter is only used as an initial one.

\subsection{Fingerprint Preprocessing}

With our method, fingerprint features are extracted from a thinned fingerprint for two reasons: First, it contains enough information to represent the uniqueness of a fingerprint. Second, in the thinned fingerprint, minutiae are more accurately detected and ridges are more efficiently tracked than its corresponding gray fingerprint. Generally, a thinned fingerprint is obtained from a series of steps including normalization, enhancement, binarization, thining, and postprocessing [17], [18]. Cheng and Tian's dyadic scale space-based fingerprint enhancement method [19], which has been applied in our AFIS for a fingerprint, is often affected by multispectrum noises. In this method, a fingerprint can be divided into a series of scale spaces with its corresponding Gaussian filter for the enhancement. The combined statistical and structural approach [18] and knowledge-based enhancement method [17] have been employed in our AFIS to binarize and postprocess the enhanced fingerprint. The fingerprint preprocessing in our AFIS is displayed in Fig. 1, where Fig. 1a is from the first fingerprint database (DB1_a) of the First International Fingerprint Verification Competition in 2000 (FVC2000) [20]; Figs. 1b, 1c, and 1d indicate the corresponding orientation image, binary image, and thinned image, respectively. 


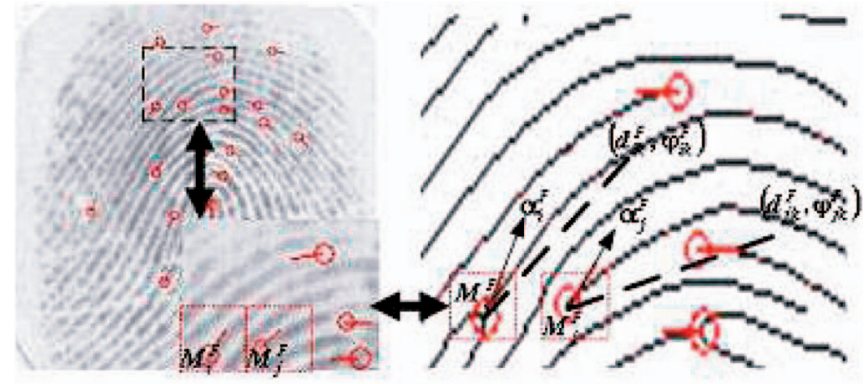

Fig. 2. Minutiae and their associated local ridge information.

\subsection{Fingerprint Representation in Our Method}

The uniqueness of a fingerprint is determined by topographic pattern of its texture structure and certain ridge anomalies termed as minutiae. A challenging task of a fingerprint matching method is to extract enough reliable features in a small memory expense. Nowadays, comprehensive features combining minutiae and ridge information have been widely used in matching and they have been performing well. However, the proposed technique includes the various texture-based features as part of the minutiae-simplex and finds fingerprint alignment. The ridge-based nearest neighborhood among minutiae is used as a feature to demonstrate the ridge-based relative relations among minutiae. This technique combines the Euclidian space-based and ridge-based relative features. In this section, comprehensive minutiae and their Euclidianbased and ridge-based relative features will be proposed to characterize the uniqueness of a fingerprint.

\subsubsection{Comprehensive Minutiae}

A fingerprint of bad quality may be too dry, too wet or the foreground area may be so narrow that no enough reliable minutiae can be detected. In some cases, two fingerprints even from the same finger fail to match for lack of common minutiae. Therefore, minutiae and associated ridge information are combined in proposed method to improve fingerprint representation.

Vector set $M^{F}=\left\{M_{i}^{F}=\left(x_{i}^{F}, y_{i}^{F}, \alpha_{i}^{F}, \beta_{i}^{F}, \varphi_{i 1}^{F}, \ldots, \varphi_{i T}^{F}, d_{i 1}^{F}\right.\right.$, $\left.\left.\ldots, d_{i T}^{F}\right) ; \quad\left|M^{F}\right| \geq i \geq 1, T \geq 2\right\}$ denote all comprehensive minutiae in fingerprint $F$, where $\left|M^{F}\right|$ is the number of the minutiae in fingerprint $F$ and $T$ denotes the number of sampled points along a ridge skeleton associated with a minutia. $M_{i}^{F}$, the $i$ th minutia, see Fig. 2, is denoted by a feature vector $\left(x_{i}^{F}, y_{i}^{F}, \alpha_{i}^{F}, \beta_{i}^{F}, \varphi_{i 1}^{F}, \ldots, \varphi_{i T}^{F}, d_{i 1}^{F}, \ldots, d_{i T}^{F}\right)\left(\left|M^{F}\right| \geq i \geq 1\right.$, $T \geq 2$ ), where:

1. $x_{i}^{F}$ and $y_{i}^{F}$ denote its coordinates.

2. $\alpha_{i}^{F}$ denotes its orientation, the angle from the horizontal axis OX to its local ridge direction in the anticlockwise direction.

3. $\beta_{i}^{F}$ denotes the local gray variance of a $16 \times 16$ area centered by $M_{i}^{F}$.

4. $\varphi_{i k}^{F}$ and $d_{i k}^{F}\left(\left|M^{F}\right| \geq i \geq 1, T \geq k \geq 1\right)$, respectively, denote the direction and distance from $M_{i}^{F}$ to the $k$ th point sampled along the ridge skeleton beginning at $M_{i}^{F}$ in the equal step.

And, the equal step is a constant pixel count between two adjacent sampled points along the skeleton. And, it is set to three times the ridge width in our study. $\varphi_{i k}^{F}$ and $d_{i k}^{F}$ are

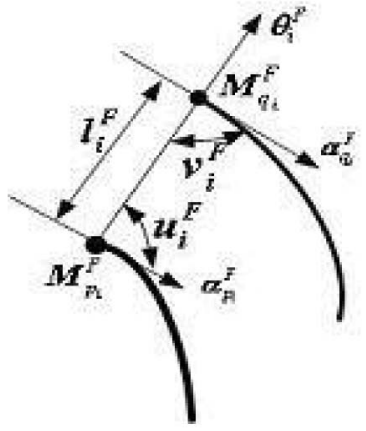

Fig. 3. Minutia-simplex $E_{i}^{F}$. Minutiae $M_{p_{i}}^{F}$ and $M_{q_{i}}^{F}$ are its two ending points; $l_{i}^{F}, \theta_{i}^{F}, u_{i}^{F}$, and $v_{i}^{F}$ are its relative features.

determined by $k$ th sampled point on the ridge associated with $M_{i}^{F}$. Therefore, $\varphi_{i k}^{F}$ and $d_{i k}^{F}$ will be affected by spurs or kinks in the skeleton. To reduce their effect on $\varphi_{i k}^{F}$ and $d_{i k}^{F}$, Luo and Tian's knowledge-based postprocessing method [17] is used to smooth fingerprint skeleton after thinning.

All $\varphi_{i k}^{F}$ and $d_{i k}^{F}$ are employed to describe the ridge information associated with $M_{i}^{F}, \beta_{i}^{F}, \varphi_{i k}^{F}$, and $d_{i k}^{F}\left(\left|M^{F}\right| \geq\right.$ $i \geq 1, T \geq k \geq 1$ ) are combined to describe local texture and ridge information of the local region associated with $M_{i}^{F}$, respectively. These features help align and distinguish input features in matching.

\subsubsection{Relative Structures among Minutiae}

In fingerprint analysis, minutiae are more abstract than fingerprint pixels. They are related to each other and attributed by (unary) properties. In other words, a fingerprint can be simply represented by minutiae constrained with their properties and relations. It is the bilateral or higher order relations that convey the contextual constraints. They play a crucial role in fingerprint matching. In this algorithm, two relative structures among minutiae are introduced as minutia-simplex and ridge-based nearest neighborhood among minutiae.

Minutia-Simplex. $n$th $(n \geq 1)$ order relative structures among minutiae combine all comprehensive minutiae as a whole. These relative structures are usually classified into unary minutia, minutia-simplex, and minutia-triplet. Minutiae have seldom been used as unary relative structures because they do not have relative features to globally represent a fingerprint. Third order relative structures, such as minutia-triplet employed in many methods [2], [3], require more computational expense though they have more robust performance in matching. Second order relative structures also have enough relative features and keep a good trade-off between computational expense and performance. Therefore, minutia-simplex, second order relative structure of minutiae is proposed in our study, as shown in Fig. 3.

Let $E^{F}=\left\{E_{i}^{F}=\left(p_{i}^{F}, q_{i}^{F}, l_{i}^{F}, \theta_{i}^{F}, u_{i}^{F}, v_{i}^{F}\right) ;\left|E^{F}\right| \geq i \geq 1\right\}$ denote the minutia-simplex set of fingerprint $F$, where:

1. $\left|E^{F}\right|$ is the size of the minutia-simplex set.

2. $p_{i}^{F}$ and $q_{i}^{F} \quad\left(\left|M^{F}\right| \geq p_{i}^{F}, q_{i}^{F} \geq 1\right)$ denote the serial numbers in the minutia set $M^{F} . M_{p_{i}}^{F}$ and $M_{q_{i}}^{F}$ are two ending minutiae of a minutia-simplex $E_{i}^{F}$ when $L_{h} \geq\left\|\left(x_{p_{i}}^{F}, y_{p_{i}}^{F}\right)-\left(x_{q_{i}}^{F}, y_{q_{i}}^{F}\right)\right\|_{2} \geq L_{l}$, where $L_{l}$ and $L_{h}$ are the lower and upper bounds of the length of a valid minutia-simplex, respectively; $\left(x_{p_{i}}^{F}, y_{p_{i}}^{F}\right)$ and $\left(x_{q_{i}}^{F}, y_{q_{i}}^{F}\right)$ are the coordinates of $M_{p_{i}}^{F}$ and $M_{q_{i}}^{F}$, respectively. 


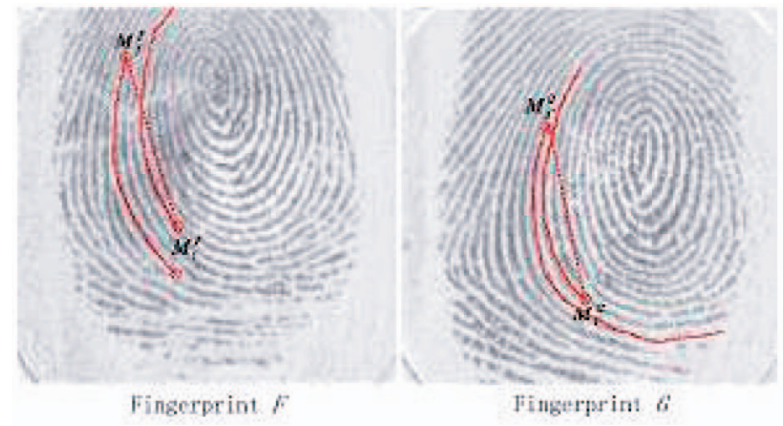

Fig. 4. Differences between ridge-based relative features. The ridgebased nearest neighborhood of $M_{i}^{F}$ and $M_{j}^{F}$ is obviously dissimilar to that of $M_{i^{\prime}}^{G}$ and $M_{j^{\prime}}^{G}$, though their Euclidean space-based relative features are similar.

Assuming that the local region centered by $M_{i}^{F}$ deforms very little, the relative features of all minutia-simplexes associated with $M_{i}^{F}$ can be linearly transformed in this local region.

3. $l_{i}^{F}=\left\|\left(x_{p_{i}}^{F}, y_{p_{i}}^{F}\right)-\left(x_{q_{i}}^{F}, y_{q_{i}}^{F}\right)\right\|_{2}$ and denotes the length of 4. the minutia-simplex.

$$
\theta_{i}^{F}=\arctan \left(\begin{array}{c}
x_{p_{i}}^{F}-x_{q_{i}}^{F} \\
y_{p_{i}}^{F}-y_{q_{i}}^{F}
\end{array}\right)
$$

denotes the direction of the minutia-simplex.

5. $u_{i}^{F}=\alpha_{p_{i}}^{F}-\theta_{i}^{F}$ and $v_{i}^{F}=\alpha_{q_{i}}^{F}-\theta_{i}^{F}$. They are derivative relative features of minutia-simplex $E_{i}^{F}$, respectively, denoting its directional differences away from $\alpha_{p_{i}}^{F}$ and $\alpha_{q_{i}}^{F}$.

Relative features of a minutia-simplex are divided into two parts, transformation-invariants and transformationvariants, which are used for local similarity measurement and alignment, respectively. Transformation-invariants $l_{i}^{F}$, $u_{i}^{F}$, and $v_{i}^{F}$ are irrelevant with linear transformation, such as translation and rotation, and can be used for direct similarity measurement if scaling is not considered. Transformationvariant $\theta_{i}^{F}$ changes with rotation and is used to model rotating input minutiae referred to the template. The size of set $E^{F},\left|E^{F}\right|$, is determined by thresholds $L_{l}$ and $L_{h} .\left|E^{F}\right|$ is much less than $\left(\begin{array}{c}\left|M^{F}\right| \\ 2\end{array}\right)$ because the distances between many minutia pairs are beyond the interval $\left[L_{l}, L_{h}\right]$. For each minutia, thresholds $L_{l}$ and $L_{h}$ are used to set a circular region in a minimum deformation, and geometric transformation of a minutia simplex is assumed to be linear. That is, the direction of a minutia-simplex in the local region is in linear relation with fingerprint rotation parameter. In the proposed method, $L_{l}$ and $L_{h}$ are set to five and 15 times ridge-width, respectively, see the Appendix.

Ridge-Based Nearest Neighborhood among Minutiae. Like a minutia-triplet, a minutia-simplex only describes the Euclidean space-based relative features among minutiae. These relative structures cannot completely explain the complex local texture and results in mismatch. For example, minutia-simplex pair $\left(M_{i}^{F}, M_{j}^{F}\right)$ and $\left(M_{i^{\prime}}^{G}, M_{j^{\prime}}^{G}\right)$ in Fig. 4 is

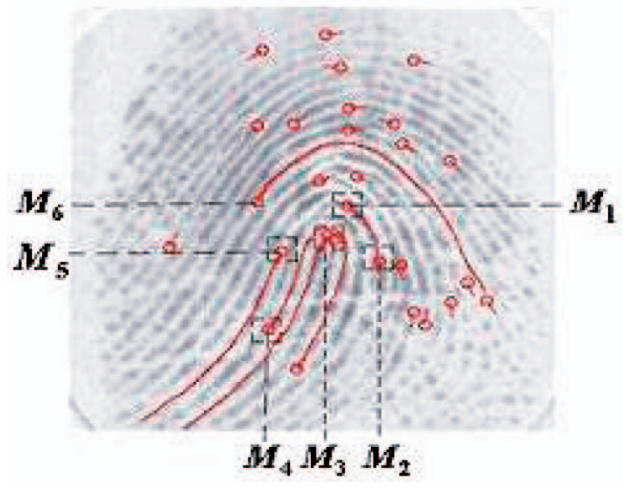

Fig. 5. Ridge-based nearest neighborhood among minutiae. 1) $M_{1}$ and $M_{2}$ are located on the same ridge. 2) There is no more than one ridge between $M_{4}$ and ridge $R_{5}$ beginning at $M_{5}$. 3) $M_{3}$ and $M_{6}$ meet neither Condition 1) nor Condition 2).

mismatched because their relative features are very similar. However, it is easier to distinguish the minutia-simplex $\left(M_{i}^{F}, M_{j}^{F}\right)$ from the minutia-simplex $\left(M_{i^{\prime}}^{G}, M_{j^{\prime}}^{G}\right)$ with their ridge-based relative features.

Let $R_{i}^{F}\left(\left|M^{F}\right| \geq i \geq 1\right)$ denote the ridge beginning at $M_{i}^{F}$ and $r_{i j}^{F}$ the ridge-count between $M_{j}^{F}$ and the ridge $R_{i}^{F}$. Set $\left\{r_{i j}^{F}|i ;| M^{F} \mid \geq j \geq 1\right\}$ denotes the ridge-based nearest neighborhood of $M_{i}^{F}$ and describes the ridge-based relative features among $M_{i}^{F}$ and other minutiae. $r_{i j}^{F}$ is more easily detected than ridge-count between two minutiae used in method [2]. In our study, $r_{i j}^{F}$ is set to 0 or 1 or 2 in three cases, respectively: 1) $r_{i j}^{F}=0$ when $M_{i}^{F}$ and $M_{j}^{F}$ are on the same ridge $R_{i}^{F}$, such as $M_{1}$ and $M_{2}$ in Fig. 5. 2) $r_{i j}^{F}=1$ when there is no more than one ridge between $M_{j}^{F}$ and $R_{i}^{F}$, like $M_{4}$ and $M_{5}$ in Fig. 5. 3) $r_{i j}^{F}=2$ when $M_{i}^{F}$ and $M_{j}^{F}$ meet neither Condition 1) nor 2), such as $M_{3}$ and $M_{6}$ in Fig. 5. The ridgebased nearest neighborhood among minutiae describes the novel ridge-based relative features among minutiae.

\subsection{Transformation Parameter Analysis}

For a randomly placed finger, it is necessary to align input minutiae to the template during matching. The alignment generally includes rotation, translation, and shearing. The alignment significantly affects the comprehensive similarity of two fingerprints. A transformation model also needs to be optimized to obtain the maximal comprehensive similarity. However, it is difficult to estimate the maximal comprehensive similarity if one doesn't know the optimal transformation model. In our study, the relationship between the comprehensive similarity and transformation model was built for estimating an optimal transformation model. The model is confirmed effective by our experimental result.

This matching algorithm is designed assuming that input and template fingerprints are captured with the same device but with little scaling deformation under the same condition. Since fingerprint matching performs well in polar coordinate, translation of the input features to the template is not used if the central point is set in advance. So, one of the most important tasks in alignment is to obtain the optimal rotation parameter.

\subsubsection{Local Comprehensive Similarity Measurement}

In some matching methods, such as Jiang and Yau's local and global structure based minutiae matching method [2], local 
similarities between relative structures are accumulated to calculate the global similarity. In our study, local similarities are also used for estimating transformation-parameters and they are calculated by coarsely comparing transformationinvariant relative features of minutiae-simplexes.

Set $S=\left\{\left(S_{i j}, I_{i j}\right) ; S_{i j} \geq 0, I_{i j}=0\right.$ or $1,\left|E^{F}\right| \geq i \geq 1,\left|E^{G}\right|$ $\geq j \geq 1\}$ denote all local comprehensive similarities between two minutia-simplex sets $E^{F}$ and $E^{G}$, where $S_{i j}$ is the local similarity between $E_{i}^{F}$ and $E_{j}^{G}$ and $I_{i j}$ is their matching order. If $I_{i j}=1, E_{i}^{F}$ and $E_{j}^{G}$ are assumed to be matched in positive order; otherwise, in reverse order.

$$
\left\{\begin{array}{l}
I_{i j}=\left(S_{i j}^{(0)}<S_{i j}^{(1)}\right) \\
S_{i j}=\max \left\{S_{i j}^{(0)}, S_{i j}^{(1)}\right\},
\end{array}\right.
$$

where the similarity measurement function $f, S_{i j}^{(0)}$, and $S_{i j}^{(1)}$ are defined as

$$
\left\{\begin{array}{l}
S_{i j}^{(0)}=f\left(\Delta, \operatorname{diff}_{0}\left(\left(E_{i}^{F}, M_{p_{i}}^{F}, M_{q_{i}}^{F}\right),\left(E_{j}^{G}, M_{q_{j}}^{G}, M_{p_{j}}^{G}\right)\right)\right) \\
S_{i j}^{(1)}=f\left(\Delta, \operatorname{diff}_{1}\left(\left(E_{i}^{F}, M_{p_{i}}^{F}, M_{q_{i}}^{F}\right),\left(E_{j}^{G}, M_{p_{j}}^{G}, M_{q_{j}}^{G}\right)\right)\right), \\
f\left(\left(\Delta_{1}, \ldots, \Delta_{n}\right),\left(x_{1}, \ldots, x_{n}\right)\right)=\frac{1}{n} \sum_{k=1}^{n} \frac{\left\|\Delta_{k}-x_{k}\right\|}{\left\|\Delta_{k}\right\|} .
\end{array}\right.
$$

In (2), $\Delta=\left(\Delta_{l}, \Delta_{\beta}, \Delta_{\beta}, \Delta_{\alpha}, \Delta_{\alpha}, \Delta_{l}, \ldots, \Delta_{l}\right)$, and $\operatorname{diff}_{1}\left(\left(E_{i}^{F}\right.\right.$, $\left.\left.M_{p_{i}}^{F}, M_{q_{i}}^{F}\right),\left(E_{j}^{G}, M_{p_{j}}^{G}, M_{q_{j}}^{G}\right)\right)$ and diff $f_{0}\left(\left(E_{i}^{F}, M_{p_{i}}^{F}, M_{q_{i}}^{F}\right),\left(E_{j}^{G}, M_{q_{j}}^{G}\right.\right.$, $\left.\left.M_{p_{j}}^{G}\right)\right)$ are defined as

$$
\left\{\begin{array}{l}
\operatorname{diff}_{1}\left(\left(E_{i}^{F}, M_{p_{i}}^{F}, M_{q_{i}}^{F}\right),\left(E_{j}^{G}, M_{p_{j}}^{G}, M_{q_{j}}^{G}\right)\right)= \\
\left(\begin{array}{l}
l_{i}^{F}-l_{j}^{G}, \beta_{p_{i}}^{F}-\beta_{p_{j}}^{G}, \beta_{q_{i}}^{F}-\beta_{q_{j}}^{G}, u_{i}^{F}-u_{j}^{G}, v_{i}^{F}-v_{j}^{G}, \\
d_{p_{i} 1}^{F}-d_{p_{j} 1}^{G}, \ldots, d_{p_{i} T}^{F}-d_{p_{j} T}^{G}, d_{q_{i} 1}^{F}-d_{q_{j} 1}^{G}, \ldots, d_{q_{i} T}^{F}-d_{q_{j} T}^{G}
\end{array}\right) \\
\operatorname{diff}_{0}\left(\left(E_{i}^{F}, M_{p_{i}}^{F}, M_{q_{i}}^{F}\right),\left(E_{j}^{G}, M_{q_{j}}^{G}, M_{p_{j}}^{G}\right)\right)= \\
\left(\begin{array}{l}
l_{i}^{F}-l_{j}^{G}, \beta_{p_{i}}^{F}-\beta_{q_{j}}^{G}, \beta_{q_{i}}^{F}-\beta_{p_{j}}^{G}, u_{i}^{F}-u_{j}^{G}-180^{\circ}, v_{i}^{F}-v_{j}^{G}-180^{\circ}, \\
d_{p_{i} 1}^{F}-d_{q_{j} 1}^{G}, \ldots, d_{p_{i} T}^{F}-d_{q_{j} T}^{G}, d_{q_{i} k}^{F}-d_{p_{j} k}^{G}, \ldots, d_{q_{i} T}^{F}-d_{p_{j} T}^{G}
\end{array}\right) .
\end{array}\right.
$$

In (3), $\Delta_{l}, \Delta_{\alpha}$, and $\Delta_{\beta}$ denote the error thresholds of distance, direction and gray variance of a minutia-simplex, respectively. They are estimated from training data sets and set to 16 pixels, $10^{\circ}, 16$, respectively, see the appendix. diff $f_{1}$ $\left(\left(E_{i}^{F}, M_{p_{i}}^{F}, M_{q_{i}}^{F}\right),\left(E_{j}^{G}, M_{p_{j}}^{G}, M_{q_{j}}^{G}\right)\right)$ is produced by matching the transformation invariant features of $\left(E_{i}^{F}, M_{p_{i}}^{F}, M_{q_{i}}^{F}\right)$ and those of $\left(E_{j}^{G}, M_{p_{j}}^{G}, M_{q_{j}}^{G}\right)$ in positive matching order. $\operatorname{diff}_{0}\left(\left(E_{i}^{F}\right.\right.$, $\left.\left.M_{p_{i}}^{F}, M_{q_{i}}^{F}\right),\left(E_{j}^{G}, M_{q_{j}}^{G}, M_{p_{j}}^{G}\right)\right)$ is generated by matching the transformation invariant features of $\left(E_{i}^{F}, M_{p_{i}}^{F}, M_{q_{i}}^{F}\right)$ and those of $\left(E_{j}^{G}, M_{q_{j}}^{G}, M_{p_{j}}^{G}\right)$ in reversed matching order. And the directional relative features of $E_{j}^{G}, \theta_{j}^{G}, u_{j}^{G}$, and $v_{j}^{G}$, are aligned as $\theta_{j}^{G}+180^{\circ}, u_{j}^{G}+180^{\circ}$, and $v_{j}^{G}+180^{\circ}$, respectively.

Deformation in fingerprints affects the similarity set $\boldsymbol{S}$ and results in mismatch. In our study, the ridge-based nearest neighborhood among minutiae is used to check all local similarities.

\subsubsection{Checking Local Similarity with Ridge-Based Nearest Neighborhood among Minutiae}

In local similarity measurement in (1), error thresholds $\Delta_{l}$, $\Delta_{\alpha}$, and $\Delta_{\beta}$ are used to counteract the influence of spurs on relative features in matching. The scheme of tolerance deviation also results in mismatch. For example, two similar local regions, being from two different fingers, are often mismatched with the Euclidean space-based relative features. In our study, the ridge-based nearest neighborhood among minutiae is used to overcome the fault by doublechecking these coarse matching results to determine local mismatch in Set $S$.

Each element in set $S$ is mismatch if $E_{i}^{F}$ and $E_{j}^{G}$ don't meet the condition described in (4), depicting the difference between the ridge-based nearest neighborhood of minutia pair $\left(M_{p_{i}}^{F}, M_{q_{i}}^{F}\right)$ and that of minutia pair $\left(M_{p_{j}}^{G}, M_{q_{j}}^{G}\right)$. If $I_{i j}=1$, indicating $E_{i}^{F}$ is matched to $E_{j}^{G}$ in positive order, $r_{p_{i} q_{i}}^{F}$ and $r_{q_{i} p_{i}}^{F}$ are compared with $r_{p_{j} q_{j}}^{G}$ and $r_{q_{j} p_{j}}^{G}$, respectively, or vice versa, if $I_{i j}=0$, indicating $E_{i}^{F}$ is matched to $E_{j}^{G}$ in reverse order, $r_{p_{i}}^{F} q_{i}$ and $r_{q_{i} p_{i}}^{F}$ are compared with $r_{q_{j} p_{j}}^{G}$ and $r_{p_{j} q_{j}}^{G}$, respectively.

$$
\left\{\begin{array}{l}
\left(\left|r_{p_{i} q_{i}}^{F}-r_{p_{j} q_{j}}^{G}\right| \leq 1\right) A N D\left(\left|r_{q_{i} p_{i}}^{F}-r_{q_{j} p_{j}}^{G}\right| \leq 1\right), \text { if } I_{i j}=1 \\
\left(\left|r_{p_{i} q_{i}}^{F}-r_{q_{j} p_{j}}^{G}\right| \leq 1\right) A N D\left(\left|r_{q_{i} p_{i}}^{F}-r_{p_{j} q_{j}}^{G}\right| \leq 1\right), \text { if } I_{i j}=0
\end{array}\right.
$$

\subsubsection{Local Transformation Parameter Estimation}

The affine transformation model is a feasible and effective model for fingerprint matching [21] and it needs three parameters, $\theta, t_{x}$, and $t_{y}$, if scaling is not considered in our study. Only the rotation parameter $\theta$ is required if the center points of two fingerprints are given and matching is done in the polar system. Based on the coarse matching results, local rotation parameter measurement is introduced in this section.

Set $\boldsymbol{\theta}=\left\{\theta_{i j} ;\left|E^{F}\right| \geq i \geq 1,\left|E^{G}\right| \geq j \geq 1\right\}$ denote all local rotation parameters calculated from all local relative structures. In our study, a local rotation parameter is represented by the direction difference between a local relative structure and its corresponding template one, which is based on the assumption that each local fingerprint region deforms very little. Equation (5) uses the mean of local directional biases of an input minutia-simplex referred to its corresponding template one as a local rotation parameter.

$$
\begin{aligned}
\theta_{i j}= & \frac{1}{2 T+2}\left[\sum_{k=1}^{T}\left[\left(\varphi_{p_{i} k}^{F}+\varphi_{q_{i} k}^{F}\right)-\left(\varphi_{p_{j} k}^{G}+\varphi_{q_{j} k}^{G}\right)\right]\right. \\
& \left.+\left(\alpha_{p_{i}}^{F}+\alpha_{q_{i}}^{F}\right)-\left(\alpha_{p_{j}}^{G}+\alpha_{q_{j}}^{G}\right)\right] .
\end{aligned}
$$

\subsubsection{Modeling the Relation between Comprehensive Similarity and Rotation Parameter}

Many methods, such as Cappelli et al.'s plastic distortion model [22], Bazen and Gerez's thin-plate spline model [23], and Senior and Bolle's equally spaced fingerprint conversion [24], are proposed to model nonlinear deformation patterns. However, these methods have their limits in fingerprint identification application systems, though they can partially solve deformation under controllable situations. For example, with the plastic distortion model [22], it is hard to obtain enough information to build the deformation model. Senior and Bolle's equally spaced fingerprint conversion [24] would fail if the compression or traction force is parallel to the local ridge orientation and the interridge space will not change. 


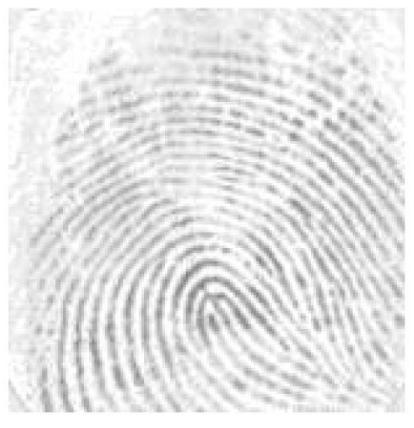

(f)

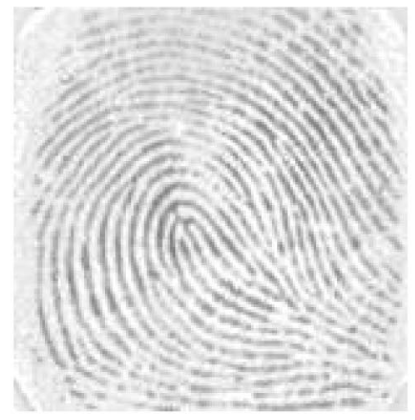

(g)
Fig. 6. Fingerprints (f) and (g) acquired from the same finger with a sensor.

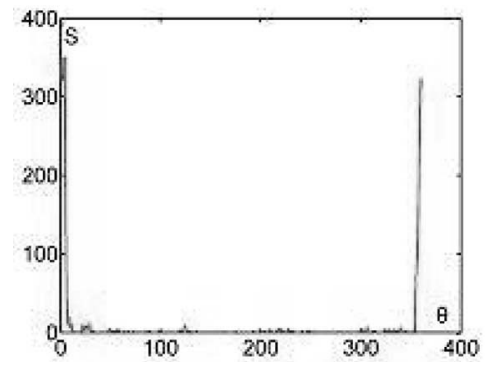

Fig. 7. Distribution of comprehensive similarity $S$ along rotation parameter $\theta$.

Bazen and Gerez's thin-plate spline model [23] is good at distinguishing the major differences from different fingerprints; however, it is weak in detecting their minor differences.

In this paper, using a series of local relative structures with little local deformation, the distribution of global comprehensive similarity along rotation parameter is modeled to calculate an optimal rotation parameter. In matching, the rotation parameter is adjusted in a small interval to reduce the impact of mismatches in the coarsely matching stage on the global rotation parameter. For local similarity set $\boldsymbol{S}$ and its corresponding local directional bias set $\theta$, their relationship is denoted with a similarity function $H(\boldsymbol{S}, \boldsymbol{\theta})$ built with (6) in terms of histogram. Fig. 7 illustrates $H(\boldsymbol{S}, \boldsymbol{\theta})$, which comes from coarse results by matching two fingerprints from the same finger, see Fig. 6 .

$$
H(S, \theta)=\sum_{i=1}^{\left|E^{F}\right|} \sum_{j=1}^{\left|E^{G}\right|} \delta_{1}\left(\theta_{i j}-\theta\right) \times S_{i j},
$$

where $\delta_{1}(x)$ is an impulsive function; if $x=0, \delta_{1}(x)=1$; otherwise, $\delta_{1}(x)=0$.

Local similarity measurement is disturbed by noises so that $H(\boldsymbol{S}, \boldsymbol{\theta})$ will be affected accordingly. A filter function, $w(x)=\frac{1}{2}\left[1-\cos \left(\frac{x \pi}{d}\right)\right](2 d+1 \geq x \geq 1)$, is used to decrease noises in $H(\boldsymbol{S}, \boldsymbol{\theta})$. Filtered $H(\boldsymbol{S}, \boldsymbol{\theta})$ is shown in Fig. 8. $H(\boldsymbol{S}, \boldsymbol{\theta})$, a periodical function, can be extended by half a period, which doesn't influence its performance. And, the histogram has only one peak in a period, where the rotation parameter is optimal. In our study, the maximum of $H(\boldsymbol{S}, \boldsymbol{\theta})$ is used to coarsely measure the gobal similarity between fingerprints $F$ and $G$.

Theoretically, the rotation parameter $\theta_{0}$, where $H\left(\boldsymbol{S}, \boldsymbol{\theta}_{0}\right)$ is the maximal, is the global optimal one, called $\theta_{m}$. It is

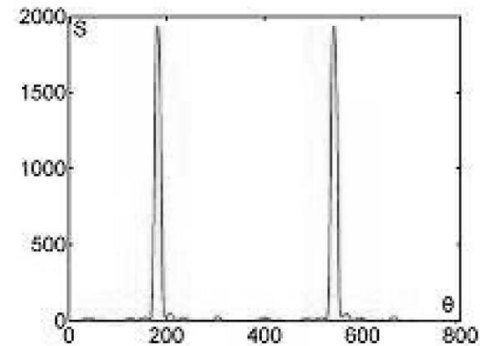

Fig. 8. Filtered distribution of similarity $S$ along rotation parameter $\theta$.

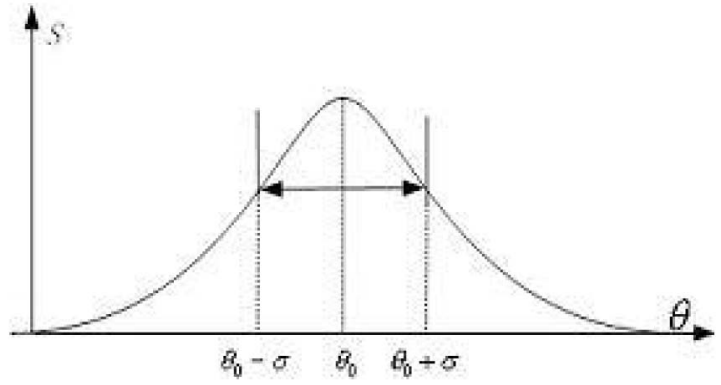

Fig. 9. Rotation parameter calculated with the geometric mean method from $H(\boldsymbol{S}, \boldsymbol{\theta})$.

calculated with geometric mean method as shown in (7) and Fig. 9, where $\theta$ is set to less than half of directional error threshold $\Delta_{\alpha}$ and $\gamma \in(0,1]$ is applied for an confidence interval of the global similarity. They are set to 5 and 0.6677 , respectively, in this method. For example, if $H(\boldsymbol{S}, \boldsymbol{\theta})$ is of normal distribution, the confidence of the global similarity is $2 \phi(\sqrt{-2 \ln \gamma})-1=0.6318$ when $\gamma=0.6667$. If $\theta_{m}$ is beyond the interval preset for the rotation transformation parameter, this matching fails. In (7), $H\left(S, \theta_{0}\right)=\max \{H(\boldsymbol{S}, \boldsymbol{\theta})\} ; h(x)=0$ if $x<0$; otherwise, $h(x)=x$.

$$
\theta_{m}=\frac{\sum_{\theta \in\left[\theta_{0}-\sigma, \theta_{0}+\sigma\right]}\left\{\theta \times h\left(H(S, \theta)-\gamma \times H\left(S, \theta_{0}\right)\right)\right\}}{\sum_{\theta \in\left[\theta_{0}-\sigma, \theta_{0}+\sigma\right]} h\left(H(S, \theta)-\gamma \times H\left(S, \theta_{0}\right)\right)} .
$$

Deformation and large-area outlier rejection will influence the Euclidean relative features and then affect the on rotation parameter estimation. For general controllable environments of small deformation and small-area outlier rejection, $\theta_{m}$ is aligned in the interval $\left[-1^{\circ},+1^{\circ}\right]$ to reduce the influences on the estimation, while, under special conditions of large-deformation and large-area outlier rejection, $\theta_{m}$ should be aligned in a larger interval and nonrigid parameter estimation will perform better.

\subsection{Fingerprint Matching}

Deformation in fingerprints may bring false local similarities in $S$ and, therefore, affects the final comprehensive similarity. Thus, the global fingerprint matching is essential after the coarse local matching if the transformation model is known. In this section, the variably sized bounding method [5] is used to double-check all local comprehensive similarities to reduce the influence of deformation in fingerprints. It consists of three steps: setting the center points, aligning the input minutiae to the template, and double-checking all the local similarities. 
First, the comprehensive similarity of a pair of minutiae is calculated from local similarity set $S$, shown in (8). The pair of minutiae with the greatest comprehensive similarity are selected as central points.

$$
\left\{\begin{array}{l}
U_{i j}=\sum_{m=1}^{\left|E^{F}\right|} \sum_{n=1}^{\left|E^{G}\right|}\left[S_{m n} \delta_{2}\left(p_{m}^{F}, q_{m}^{F}, p_{n}^{G}, q_{n}^{G}, i, j, I_{m n}\right)\right] \\
\delta_{2}(x, y, u, v, a, b, \lambda)=\left\{\begin{array}{l}
1, \text { if }(a, b) \in\{(x, u),(y, v)\} \wedge(\lambda=1) \\
1, \text { if }(a, b) \in\{(x, v),(y, u)\} \wedge(\lambda=0) \\
0, \text { otherwise. }
\end{array}\right.
\end{array}\right.
$$

Let $U=\left\{U_{i j} ;\left|M^{F}\right| \geq i \geq 1,\left|M^{G}\right| \geq j \geq 1\right\}$ denote the set of all comprehensive similarities of minutia pairs, where $U_{i j}$ is the sum of local similarities of all minutia-simplex pairs in $\left\{\left(E_{m}^{F}, E_{n}^{G}\right) \mid\left(\left(i=p_{m}^{F}\right.\right.\right.$ or $\left.q_{m}^{F}\right)$ and $\left(j=p_{n}^{G}\right.$ or $\left.\left.\left.q_{n}^{G}\right)\right)\right\}$, and denotes the comprehensive similarity between $M_{i}^{F}$ and $M_{j}^{G}$. $\left(O_{F}, O_{G}\right)=\left(\left(x_{o_{i}}^{F}, y_{o_{i}}^{F}\right),\left(x_{o_{j}}^{G}, y_{o_{j}}^{G}\right)\right)$ is selected as the center point in alignment, where $U_{o_{i} o_{j}}=\max _{(i, j)}\left\{U_{i j}\right\}\left(\left|M^{F}\right| \geq i, o_{i} \geq 1\right.$, $\left.\left|M^{G}\right| \geq j, o_{j} \geq 1\right)$. In (8), function $\delta_{2}$ is used to judge whether a minutia-simplex pair is associated with the minutia pair $\left(M_{i}^{F}, M_{j}^{G}\right)$.

Second, all minutiae in sets $M^{F}$ and $M^{G}$ are transformed into their corresponding polar systems referring to their central points, $O_{F}$ and $O_{G}$, respectively. Let $V=\left\{V_{i j} ;\left|M^{F}\right| \geq\right.$ $\left.i \geq 1\left|M^{G}\right| \geq j \geq 1\right\}$ denote all similarities of aligned minutia pairs, where $V_{i j}$ denotes that of $M_{i}^{F}$ and $M_{j}^{G}$, see (9).

$$
\left\{\begin{array}{l}
V_{i j}=f\left(\Delta_{m_{i j}}, \operatorname{diff}\left(M_{i}^{F}, M_{j}^{G}\right)\right), \text { if } U_{i j}>0 \\
\Delta_{m_{i j}}=\left(\Delta_{\alpha}, \ldots, \Delta_{\alpha}, b\left(l_{i} / r_{0}, r_{s}, r_{l}\right), b\left(\alpha_{0} / l_{i}^{2}, \alpha_{s}, \alpha_{l}\right)\right) \\
\operatorname{diff}\left(M_{i}^{F}, M_{j}^{G}\right)=\left(\ldots, \varphi_{i k}^{F}-\varphi_{j k}^{G}-\theta_{m}, \ldots, \alpha_{i}^{F}-\alpha_{j}^{G}-\theta_{m},\right. \\
\left.\quad \rho_{i}^{F}-\rho_{j}^{G}, \vartheta_{i}^{F}-\vartheta_{j}^{G}-\theta_{m}\right) .
\end{array}\right.
$$

If $V_{i j} \leq 0, M_{i}^{F}$ and $M_{j}^{G}$ are mismatched. In (9), $b\left(x, x_{0}, x_{1}\right)$ is a step function proposed in the variably sized bounding method [5] shown in (10). The positional parameters $r_{0}, r_{s}$, and $r_{l}$ and direction parameters $a_{0}, a_{s}$, and $a_{l}$ decide the size of the variably sized bounding box; $\left(\rho_{i}^{F}, \vartheta_{i}^{F}\right)$ and $\left(\rho_{j}^{G}, \vartheta_{j}^{G}\right)$ are the coordinates of $M_{i}^{F}$ and $M_{j}^{G}$ in polar systems, respectively; $\Delta_{m_{i j}}$ is the vector of error thresholds of aligned minutia-pair $M_{i}^{F}$ and $M_{j}^{G}$; and $\operatorname{diff}\left(M_{i}^{F}, M_{j}^{G}\right)$ denotes the transformationvariant feature differences of $M_{i}^{F}$ referred to $M_{j}^{G}$.

$$
b\left(x, x_{0}, x_{1}\right)= \begin{cases}x_{0}, & \text { if } x<x_{0} \\ x, & \text { if } x_{0} \leq x<x_{1} \\ x_{1}, & \text { if } x \geq x_{1} .\end{cases}
$$

Finally, all local similarities in $S$ are double-checked with (9) in two cases. If $I_{m n}=1$, indicating that $E_{m}^{F}$ and $E_{n}^{G}$ match in positive order, $S_{m n}$ is invalid when $V_{p_{m}^{F} p_{n}^{G}} \leq 0$ and $V_{q_{m}^{F} q_{n}^{G}} \leq 0$. If $I_{m n}=0$, indicating that $E_{m}^{F}$ and $E_{n}^{G}$ match in inverse order, $S_{m n}$ is invalid when $V_{p_{m}^{F} p_{n}^{G}} \leq 0$ and $V_{q_{m}^{F} q_{n}^{G}} \leq 0$.

The revised local similarity set $S^{\prime}=\left\{\left(S_{m n}^{\prime}, I_{m n}^{\prime}\right) ;\left|E^{F}\right| \geq\right.$ $\left.i \geq 1,\left|E^{G}\right| \geq j \geq 1\right\}$ is performed as the final matching step to measure the similarity between fingerprints $F$ and $G$. $\sum_{\left(S_{m n}^{\prime}, I_{m n}^{\prime}\right) \in S^{\prime}} S_{m n}^{\prime}, \sum_{V_{i j} \in V} V_{i j},\left|S^{\prime}\right|$, and $|V|$ are four final indices for global comprehensive similarity measurement between fingerprint $F$ and $G$. They made it possible to judge whether

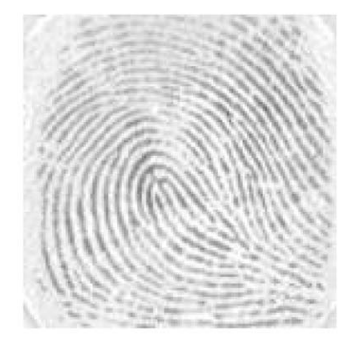

(a1)

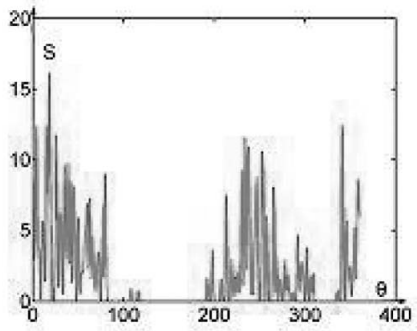

(b)

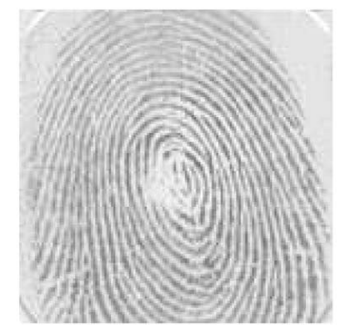

(a2)

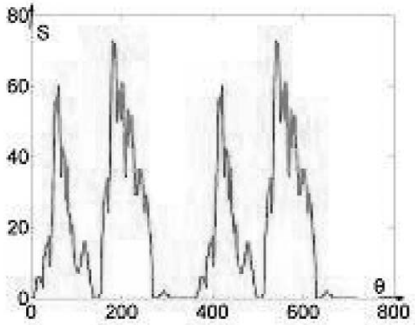

(c)
Fig. 10. A pair of fingerprints selected from the first group and their $H(S, \theta)$. (a1) and (a2) Two from two different fingers, (b) their $H(S, \theta)$, and (c) their filtered $H(S, \theta)$.

two fingerprints come from the same finger in quantity. To reduce the effects of false local similarities in $S$ on parameter estimation, $\theta_{m}$ is changed in the interval $\left[-1^{\circ},+1^{\circ}\right]$ to make matching more robust. The above matching process is also looped three times to obtain an optimal team of performance indices $\sum_{\left(S_{m n}^{\prime}, I_{m n}^{\prime} \in S^{\prime}\right)} S_{m n}^{\prime}, \sum_{V_{i j} \in V} V_{i j},\left|S^{\prime}\right|$, and $|V|$ as the final result in terms of the sum rule.

\section{Results}

Experiments were performed over the fingerprint databases provided by the First International Fingerprint Verification Competition in 2000 (FVC2000) [20], the Second International Fingerprint Verification Competition in 2002 (FVC2002) [25], and the Third International Fingerprint Verification Competition in 2004 (FVC2004) [26]. Our experiments checked the validity of $H(S, \theta)$, analyzed the rotation parameter, and evaluated the final matching performance.

\subsection{Validation of $H(S, \theta)$ in Verification}

Two sets of experiments were conducted to evaluate the performance of $H(S, \theta)$. One hundred pairs of fingerprints were selected from different fingers. In the first group of 50 pairs, two fingerprints in each pair were not similar as shown in Fig. 10a1 and Fig. 10a2. In the second group of 50 pairs, two fingerprints in each pair were similar, see Fig. 11a1 and Fig. 11a2. Fig. 10c and Fig. 11c showed that the filtered $H(S, \theta)$ of a pair of fingerprints, selected either from the first group or the second, has the characters as: 1) the comprehensive similarity is of random distribution along the rotation parameter in a period, which is caused by mismatches produced by spurs or deformation in fingerprints, and 2) both the maximum and sum of $H(S, \theta)$ are small. Compared with the shape of Fig. 10, that of Fig. 11 is more regular because two fingerprints in Fig. 11a1 and Fig. 11a2 were more similar, which resulted in more local matches. 


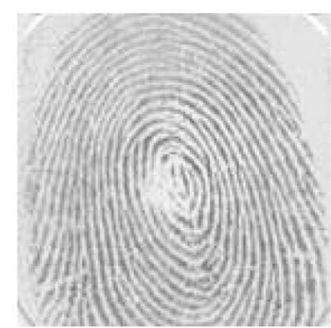

(a1)

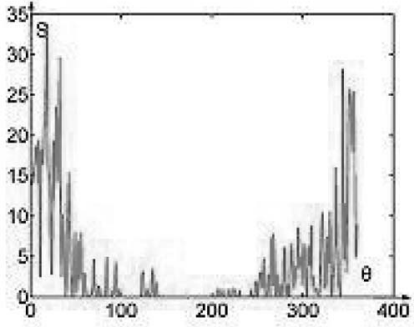

(b)

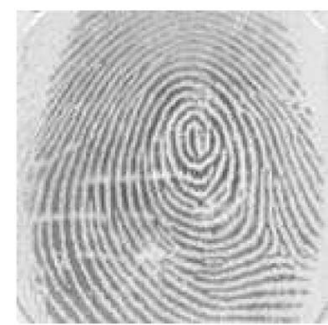

(a2)

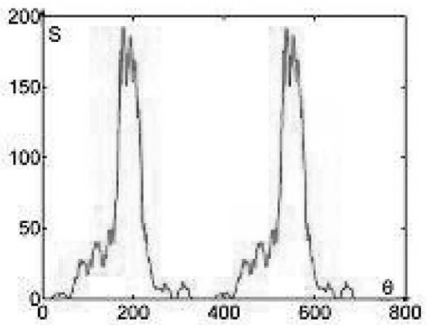

(c)
Fig. 11. A pair of fingerprints selected from the second group and their $H(S, \theta)$. (a1) and (a2) Two from two different fingers, (b) their $H(S, \theta)$, and (c) their filtered $H(S, \theta)$.

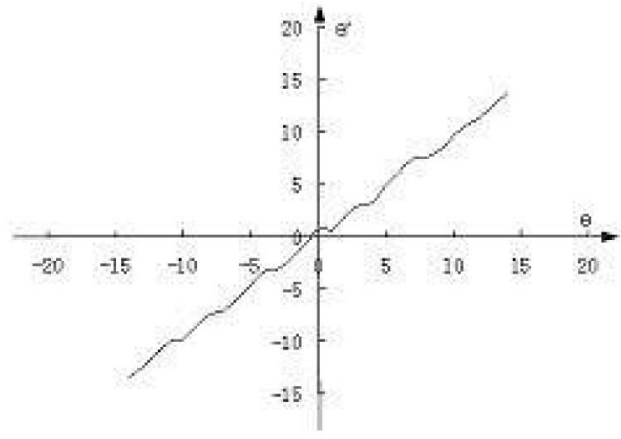

Fig. 12. The distribution of rotation parameter biases between $\theta^{\prime}$ and $\theta$.

\subsection{Validation of the Rotation Transformation Parameter Estimation}

Two experiments were conducted to evaluate the performance of the $H(S, \theta)$ in rotation parameter estimation and alignment. The first experiment was performed over 20 fingerprints randomly selected from 12 fingerprint databases of FVC2000, FVC2002, and FVC2004. For fingerprint $F$, its transformed fingerprint $F^{\prime}$ was produced by rotating $F$ with an angle $\theta$, which changed from $-15^{\circ}$ to $15^{\circ}$, referring to its central point. And, rotation parameters $\theta^{\prime}$ between $F$ and $F^{\prime}$ were estimated from their $H(S, \theta)$. These results are shown in Fig. 12. The mean and standard deviations of the absolute errors $\left|\theta-\theta^{\prime}\right|$, were $0.42^{\circ}$ and 0.26 , respectively.

The second experiment was conducted to evaluate the global alignment of input minutiae with the estimated rotation parameter. In this experiment, 50 groups of fingerprints were randomly selected from the 12 fingerprint databases. Each group contained three fingerprints from the same finger. For each group of fingerprints, as shown in Figs. 13a, 13b, and 13c, the minutiae in the second and third fingerprints were mapped onto the first fingerprint with its corresponding estimated rotation parameter $\theta_{m}$, see Fig. 13c.

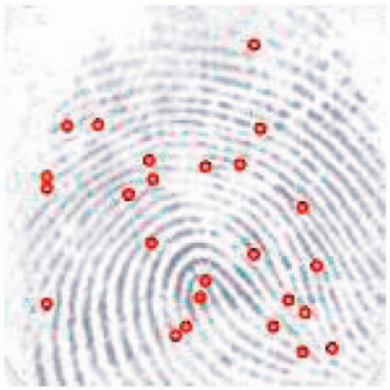

(a)

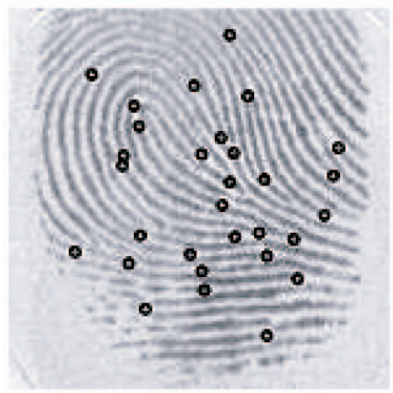

(c)

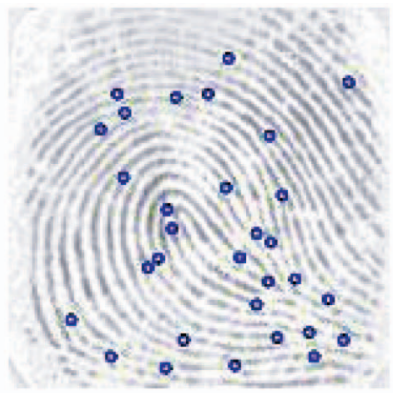

(b)

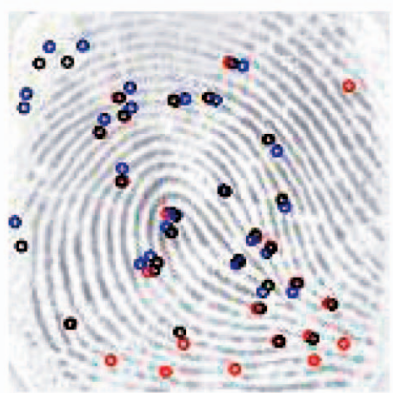

(d)
Fig. 13. Performance of the alignment. Minutiae in fingerprints (b) and (c) were mapped onto fingerprint (a), respectively, see (d).

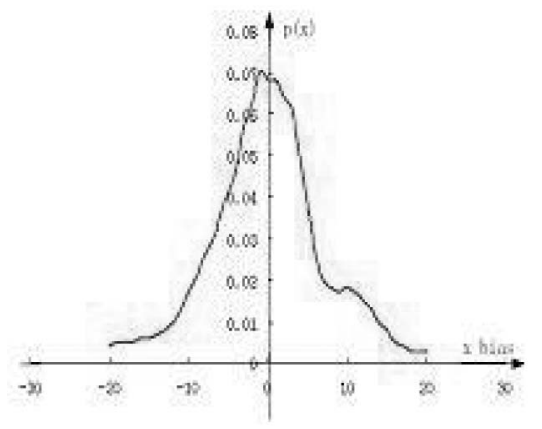

(a)

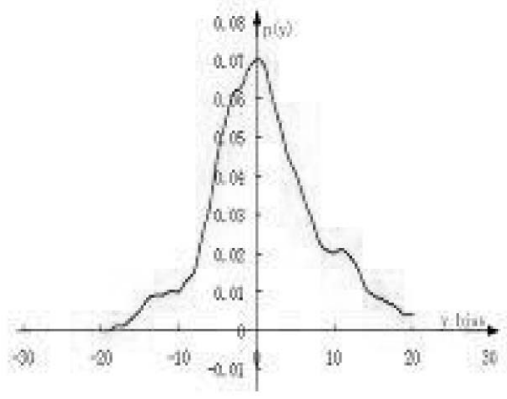

(b)

Fig. 14. Distributions of position biases of aligned input minutiae to their corresponding template ones. (a) Distribution of $x$ biases. (b) Distribution of $y$ biases. 
TABLE 1

Results of Our New Method over the Four Databases of FVC2000

\begin{tabular}{|c|c|c|c|c|c|c|c|}
\hline $\begin{array}{c}\text { Database } \\
(\text { FVC2000) }\end{array}$ & $\begin{array}{c}\text { EER } \\
(\%)\end{array}$ & $\begin{array}{c}\text { EER* } \\
(\%)\end{array}$ & $\begin{array}{c}\text { ZeroFMR } \\
(\%)\end{array}$ & $\begin{array}{c}\text { ZeroFNMR } \\
(\%)\end{array}$ & $\begin{array}{c}\text { Rej_Match } \\
(\%)\end{array}$ & $\begin{array}{c}\text { Rej_Enroll } \\
(\%)\end{array}$ & $\begin{array}{c}\text { AE\&MT } \\
(\mathrm{S})\end{array}$ \\
\hline DB1_a & 1.79 & 1.79 & 4.39 & 100 & 0.000 & 0.000 & 0.82 \\
\hline DB2_a & 0.99 & 0.99 & 2.49 & 100 & 0.000 & 0.000 & 1.10 \\
\hline DB3_a & 3.54 & 3.54 & 9.74 & 100 & 0.000 & 0.000 & 0.85 \\
\hline DB4_a & 1.64 & 1.64 & 5.05 & 100 & 0.000 & 0.000 & 0.87 \\
\hline
\end{tabular}

TABLE 2

Results of Our New Method over the Four Databases of FVC2002

\begin{tabular}{|c|c|l|l|l|l|l|l|}
\hline $\begin{array}{c}\text { Database } \\
(\text { FVC2002) }\end{array}$ & $\begin{array}{c}\text { EER } \\
(\%)\end{array}$ & $\begin{array}{c}\text { FMR100 } \\
(\%)\end{array}$ & $\begin{array}{c}\text { FMR1000 } \\
(\%)\end{array}$ & $\begin{array}{c}\text { ZeroFMR } \\
(\%)\end{array}$ & $\begin{array}{c}\text { Rej_Match } \\
(\%)\end{array}$ & $\begin{array}{c}\text { Rej_Enroll } \\
(\%)\end{array}$ & $\begin{array}{c}\text { AE\&MT } \\
(\mathrm{S})\end{array}$ \\
\hline DB1_a & 1.963 & 2.500 & 4.000 & 5.036 & 0.000 & 0.000 & 0.83 \\
\hline DB2_a & 1.110 & 1.250 & 1.964 & 4.286 & 0.000 & 0.000 & 1.20 \\
\hline DB3_a & 4.312 & 7.143 & 10.250 & 13.107 & 0.000 & 0.000 & 0.73 \\
\hline DB4_a & 2.772 & 3.429 & 6.071 & 7.679 & 0.000 & 0.000 & 0.83 \\
\hline
\end{tabular}

TABLE 3

Results of Our New Method over the Four Databases of FVC2004

\begin{tabular}{|c|c|c|l|l|l|l|l|}
\hline $\begin{array}{c}\text { Database } \\
(\text { FVC2004 })\end{array}$ & $\begin{array}{c}\text { EER } \\
(\%)\end{array}$ & $\begin{array}{c}\text { FMR100 } \\
(\%)\end{array}$ & $\begin{array}{c}\text { FMR1000 } \\
(\%)\end{array}$ & $\begin{array}{c}\text { ZeroFMR } \\
(\%)\end{array}$ & $\begin{array}{c}\text { Rej_Match } \\
(\%)\end{array}$ & $\begin{array}{c}\text { Rej_Enroll } \\
(\%)\end{array}$ & $\begin{array}{c}\text { AE\&MT } \\
(\mathrm{S})\end{array}$ \\
\hline DB1_a & 9.335 & 18.500 & 25.036 & 30.286 & 0.000 & 0.000 & 0.81 \\
\hline DB2_a & 7.345 & 13.393 & 16.607 & 19.893 & 0.000 & 0.000 & 0.76 \\
\hline DB3_a & 8.529 & 13.107 & 16.536 & 22.536 & 0.000 & 0.000 & 1.02 \\
\hline DB4_a & 2.719 & 4.214 & 5.571 & 7.000 & 0.000 & 0.000 & 0.78 \\
\hline
\end{tabular}

Fig. 14 illustrates the distribution of positional differences between aligned input minutiae and their corresponding template ones.

\subsection{Matching Performance Analysis}

To evaluate the overall matching performance of this method, a series of experiments were conducted over 12 fingerprint databases of FVC2000, FVC2002, and FVC2004, see Tables 1, 2, 3, Figs. 15, 16, and 17. In Fig. 15, the four solid lines denoted Receiving Operating Curves (ROCs) drawn in log-log scales of this method over the four databases of FVC2000, respectively, the " + " lines denoted ones over the four databases of FVC2002, and the "O" lines denoted ones over the four databases of FVC2004. Tables 1,2, and 3 described the performance of this method over the 12 databases with some performance indices provided by FVC2000 and FVC2002. This new method obviously outperformed the previous work, variably bounded box-based matching method [5], see Fig. 16. In the two methods, the same fingerprint enhancement method, Cheng and Tian's dyadic scale space-based fingerprint enhancement method [19] and Luo and Tian's knowledge-based postprocessing method [17] were applied for fingerprint preprocessing and comprehensive minutia detection were employed in matching. Their performance evaluated over DB1_a of FVC2000 demonstrated the effectiveness of the new matching method.

To judge whether ridge-based feature and ridge information were helpful for fingerprint matching, the method without ridge-based relative features or ridge information, denoted by GCS_NN, was compared with the method having only ridge information, called GCS_NR, and the method with ridge-based relative features and ridge information, called GCS_GR over the four fingerprint databases DB1_a of FVC2002, DB1_a, DB2_a, and DB4_a of
2004, respectively. Among the 12 fingerprint databases of FVC2000, FVC2002, and FVC2004, the overall fingerprint quality of DB1_a and DB2_a of 2004 is the worst, while DB1_a of FVC2002 and DB4_a of FVC2004 are the better databases. The differences among the four fingerprint databases illustrated by Fig. 17 and Table 4 proved that ridge-based relative features and ridge information were available in fingerprint matching and those features help decrease the scores of impostor matches. For example, there is a step jump at FMR close to -4 of ROC, such as line " $\mathrm{o}^{\text {" in }}$ Fig. $17 \mathrm{~b}$, if the scores of impostor matches are too great.

We noted that our method outperformed Tico and Kuosmanen's matching method with an orientation-based minutia descriptor [14] over the first and second databases of FVC2000 and Teoh et al.'s matching method with integrated wavelet and Fourier-Mellin invariant transformation [10] over the four databases of FVC2002. The good performance of GCS_GR over the databases of FVC2000, FVC2002, and FVC2004 was contributed to by the following aspects: 1) Minutia was replaced by minutia-simplex and minutiasimplex had more relative features to represent a fingerprint, 2) the ridge-based nearest neighborhood among minutiae was employed to check coarse matching, and 3) the rotation parameter was calculated in terms of the histogram.

\subsection{Template Size Analysis}

In our method, the memory expense of a comprehensive minutia depicted by a minutiae and its associate ridge information is 8 bytes if $T$, the number of sampled points along a ridge skeleton, is set to 2 . $T$ can be reasonably modified according to the memory requirement of an AFIS. Empirically, the average memory expense used to present ridge-based nearest neighborhood is no more than 4.5 bytes. 


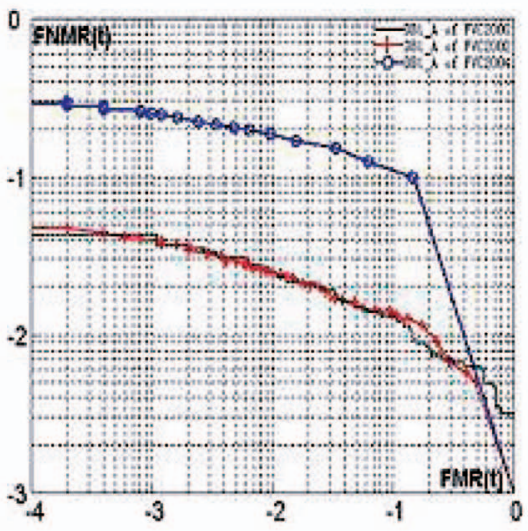

(a)

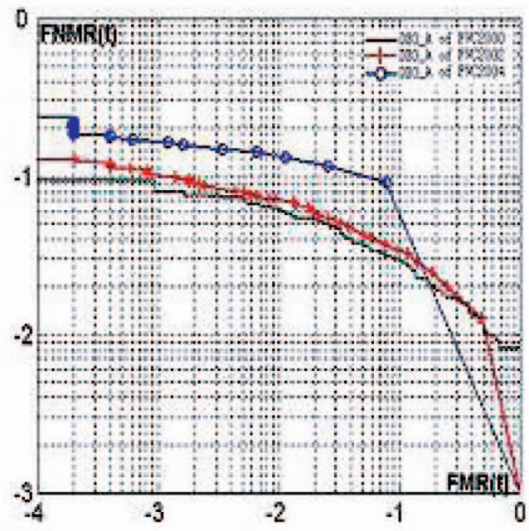

(c)

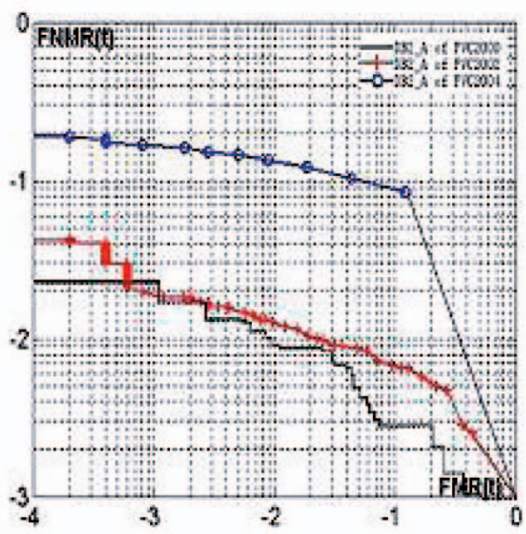

(b)

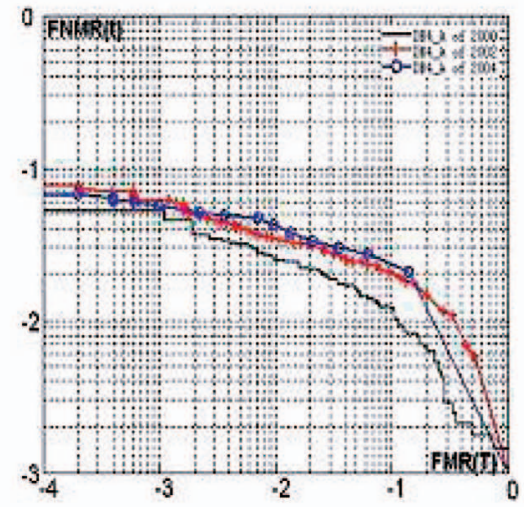

(d)

Fig. 15. ROCs (drawn in log-log scales) of our new method over the 12 databases of FVC2000, FVC2002, and FVC2004, respectively.

Therefore, the mean memory expense is 12.5 bytes for a comprehensive minutiae representation. Minutia-simplexes don't influence the memory expense for storage.

The number of minutiae varies in different fingerprints, but a good one contains 60-80 minutiae [9]. Assuming there are 80 minutiae in a fingerprint, then 1,000 bytes is more than enough for a fingerprint presentation. In different methods,

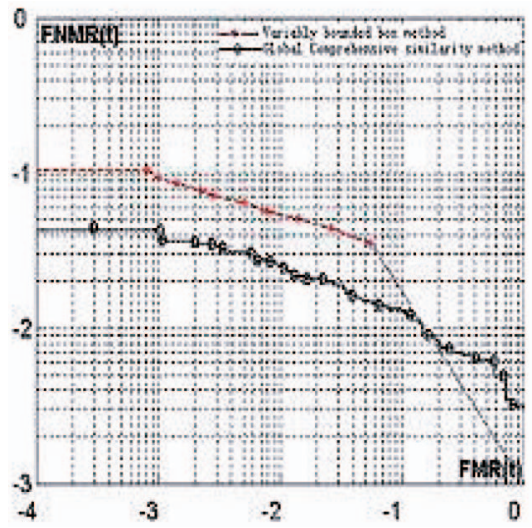

Fig. 16. Performance difference between the variably bounded box method and the new method over DB1_a of FVC2000. The "*" line and the "o" line denote their ROCs (drawn in log-log scales) over the database, respectively. template size varies. For a method based on comprehensive feature composing of minutiae and ridge information, its template size is generally more than $1 \mathrm{k}$ bytes. For example, for Ross et al.'s hybrid fingerprint method [13] and Tico and Kuosmanen's orientation-based minutia descriptor [14], more than $1 \mathrm{~K}$ bytes are required to describe the features of a fingerprint. For methods based on global features, their template sizes may be larger, such as Sujan and Mulqueen's space invariant transforms based fingerprint identification method [11]. For methods using only minutiae, such as Gold and Rangarajan's graph matching method [4], their template sizes are no more than 500 bytes, but their performance is compromised.

\section{Conclusion, Discussion, ANd FURTher Work}

A new fingerprint matching method based on global comprehensive similarity is introduced in this paper with two novel techniques. First, a minutia-simplex and the ridgebased nearest neighborhood among minutiae are performed to represent two relative structures among minutiae in different directions. Second, $H(S, \theta)$ is defined to model the relationship between transformation parameters and comprehensive similarity in terms of histogram. From this histogram, an optimal rotation parameter is estimated for alignment. Our method works well over the fingerprint databases of FVC2000, FVC2002, and FVC2004, and it can be 


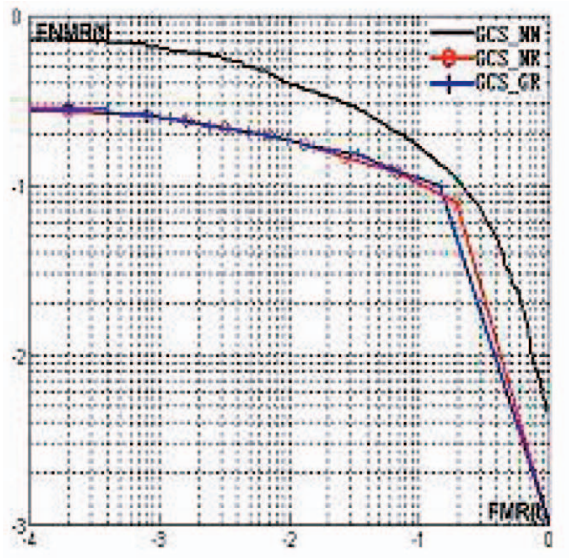

(a)

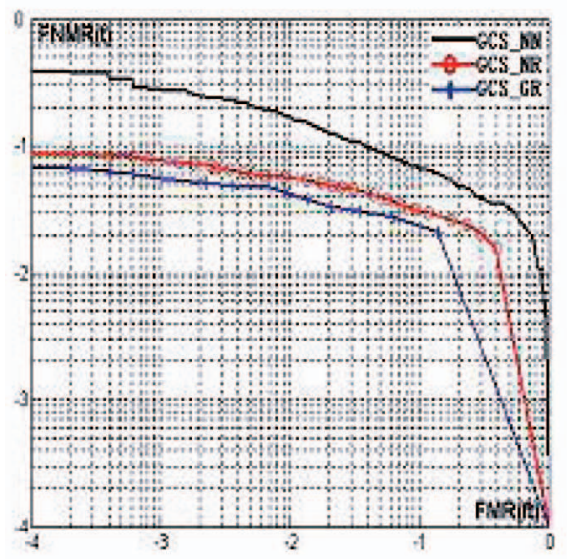

(c)

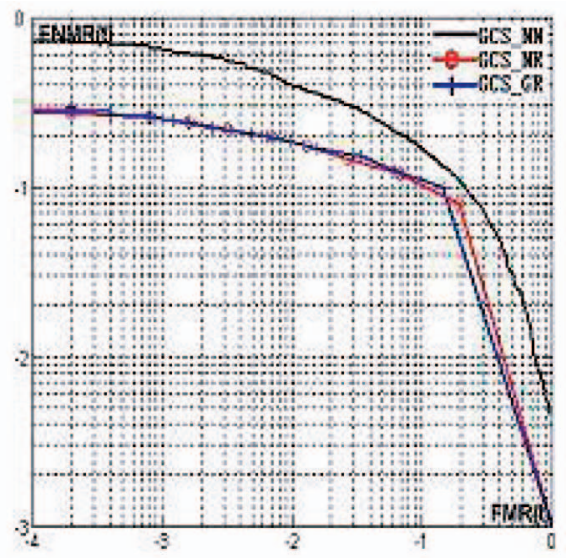

(b)

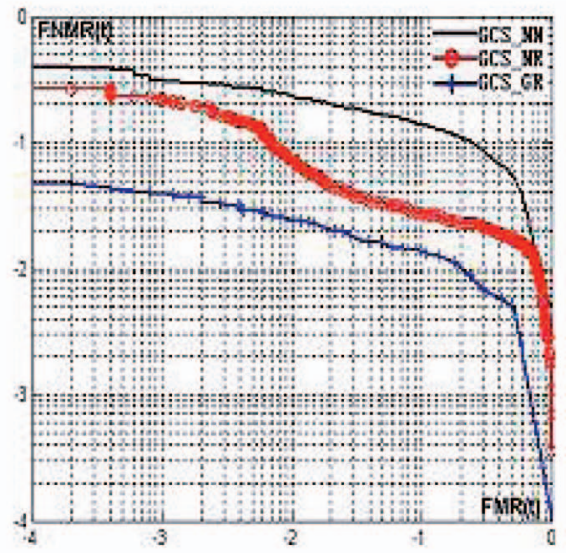

(d)

Fig. 17. Performance differences among GCS_NN, GCS_NR, and GCS_GR over four databases. (a) Performance difference over DB1_a of FVC2004. (b) Performance difference over DB2_a of FVC2004. (c) Performance difference over DB4_a of FVC2004. (d) Their performance difference over DB1_a of FVC2002.

TABLE 4

Performance Differences among GCS_NN, GCS_NR, and GCS_GR over Four Databases

\begin{tabular}{|c|r|r|r|}
\hline Database & EER of GCS_NN(\%) & EER of GCS_NR(\%) & EER of GCS_GR(\%) \\
\hline DB1_a of FVC2004 & 13.707 & 10.028 & 9.335 \\
\hline DB2_a of FVC2004 & 12.773 & 8.876 & 7.345 \\
\hline DB4_a of FVC2004 & 7.432 & 3.813 & 2.719 \\
\hline DB1_a of FVC2002 & 3.528 & 2.363 & 1.963 \\
\hline Average of |EER of GCS_NR - EER of GCS_NN|: 3.090 \\
Average of |EER of GCS_GR - EER of GCS_NR|: 0.930 \\
\hline
\end{tabular}

applied to a memory-limited AFIS owing to its less than $1 \mathrm{~K}$ byte template size. However, this method is sensitive to the quality of the fingerprint. The quality of fingerprints affects the reliability of minutiae, rotation parameter, center points, and, therefore, affects matching performance. As shown in Fig. 17a, the ridge-based relative feature has limited ability to improve fingerprint matching performance in badquality fingerprints. For example, 99_5.tif in the DB2_a of FVC2004 is a bad-quality fingerprint with no more than four genuine minutiae detected in our method. In another case, 85_1.tif and 85_8.tif in DB1_a of FVC2004 have a small common region of good quality. As a result, less than three genuine minutiae are matched.
We will continue our investigations to improve the method in minimizing false match, which occasionally occurs under the condition of large deformation in fingerprints and very poor-quality fingerprints. Based on Chen et al.'s registration pattern inspection method [27], the adaptive matching template will be tested in fingerprint matching to reduce the impacts of deformation in a fingerprint. Global pattern and features, as well as a hybrid matching technique will be investigated to reduce the sensitivity of poor quality fingerprint. Additionally, we will study the technique that employs a multiresolution search strategy to calculate the optimal transformation. 


\section{APPENDIX}

\section{THRESHOLD ESTIMATION}

In the proposed method, the thresholds, i.e., $\Delta_{l}, \Delta_{\alpha}, \Delta_{\beta}$ (the error thresholds of distance, direction, and gray variance of a minutia-simplex), $L_{l}$ and $L_{h}$ (the lower and upper bounds of the distant attribute in a valid minutia-simplex), are estimated from a training fingerprint data set that consists of $N$ pairs of fingerprints with the following steps:

Step 1. Build a set of transformation-irrelevant feature offset vectors of minutiae-simplex. For each pair of fingerprints, $F_{k}$ and $G_{k}$, label their corresponding minutia pairs and push them into set $\left\{\left(M_{i}^{F_{k}}, M_{i}^{G_{k}}\right)\right\}$, then, randomly select two minutia pairs $\left(M_{i}^{F_{k}}, M_{i}^{G_{k}}\right)$ and $\left(M_{j}^{F_{k}}, M_{j}^{G_{k}}\right)$ from set $\left\{\left(M_{i}^{F_{k}}\right.\right.$, $\left.\left.M_{i}^{G_{k}}\right)\right\}$ to build two minutia-simplexes $E_{m}^{F_{k}}$ and $E_{m}^{G_{k}}$ without distant constraint, and then compare the transformationirrelevant features of $E_{m}^{F_{k}}$ and $E_{m}^{G_{k}}$ to get the offsets described by vector $\left(d_{m}, d l_{m}, d \alpha_{m}, d \beta_{m}\right)$, where $d_{m}=\min \left\{l_{m}^{F_{k}}, l_{m}^{G_{k}}\right\}$, $d \alpha_{m}=\left(\left|u_{m}^{F_{k}}-u_{m}^{G_{k}}\right|+\left|v_{m}^{F_{k}}-v_{m}^{G_{k}}\right|\right) / 2, d l_{m}=\left|l_{m}^{F_{k}}-l_{m}^{G_{k}}\right|$, and $d \beta_{m}=\left(\left|\beta_{i}^{F_{k}}-\beta_{i}^{G_{k}}\right|+\left|\beta_{j}^{F_{k}}-\beta_{j}^{G_{k}}\right|\right) / 2$; finally, put the vector into offset vector set $D_{k}$. All combinations of two elements out of the set $\left\{\left(M_{i}^{F_{k}}, M_{i}^{G_{k}}\right)\right\}$ are used to create offset vectors and added into set $D_{k}$.

All pairs of fingerprint are examined by the same method to produce the corresponding offset vector sets $D_{k}(N \geq k \geq 1)$. These sets are united as $D=D_{1} \cup D_{2} \cup \ldots \cup D_{N}$.

Step 2. Estimate thresholds $\Delta_{l}, \Delta_{\alpha}$, and $\Delta_{\beta}$. Histograms of $d l, d \alpha$, and $d \beta$ built with set $D$ are denoted as $H_{d l}(d l), H_{\alpha}(d \alpha)$, and $H_{d \beta}(d \beta)$, respectively. According to these histograms, thresholds $\Delta_{l}, \Delta_{\alpha}, \Delta_{\beta}$ and their corresponding threshold space $T=\left\{(d l, d \alpha, d \beta) \mid d l \leq \Delta_{l} ; d \alpha \leq \Delta_{\alpha} ; d \beta \leq \Delta_{\beta}\right\}$ are calculated when $\frac{\left|D^{\prime}\right|}{|D|} \geq \eta_{1}$, where $D^{\prime}=\left\{\left(d_{m}, d l_{m}, d \alpha_{m}, d \beta_{m}\right)\right.$ $\left.\mid\left(d l_{m}, d \alpha_{m}, d \beta_{m}\right) \in T\right\}$.

Step 3. Estimate the lower and upper bounds of the distant feature of a minutia-simplex. First, build the histogram of the distant feature of minutia-simplex, called as $H_{d}(d)$ with set $D^{\prime}$; then, find $d_{o}$ and an interval $\left[L_{l}, L_{h}\right]$ on the condition that $H_{d}\left(L_{l}\right)=H_{d}\left(L_{h}\right), \frac{\left|D^{\prime \prime}\right|}{\left|D^{\prime}\right|} \geq \eta_{2}, H_{d}\left(d_{o}\right)=$ $\max \left\{H_{d}(d)\left(L_{h} \geq d_{o} \geq L_{l}\right)\right.$, where $D^{\prime \prime}=\left\{D_{m}^{\prime \prime}=\left(d_{m}, d l_{m}\right.\right.$, $\left.d \alpha_{m}, d \beta_{m}\right) \mid D_{m}^{\prime \prime} \in D$ and $\left.d_{m} \in\left[L_{l}, L_{h}\right]\right\}$. Statistically, if $\left|D^{\prime}\right|$ is enough large, $H_{d}(d)$ is of normal distribution. In threshold estimation, both $\eta_{1}$ and $\eta_{2}$ are so great that set $D^{\prime \prime}$ has enough elements, representing the differences among minutia-simplex pairs, to calculate the similarity between two fingerprints.

\section{ACKNOWLEDGMENTS}

This research is aided by the National Science Fund for Distinguished Young Scholars of China under Grant No. 60225008, the Key Project of the National Natural Science Foundation of China under Grant No.60332010, and the National Natural Science Foundation of China under Grant No. 60303022. The authors would like to thank Professor Anil K. Jain and Professor Ying Liu for their careful proofreading.

\section{REFERENCES}

[1] S.Z. Li, Markov Random Field Modeling in Image Analysis. SpringerVerlag, 2001.

[2] X.D. Jiang and W.Y. Yau, "Fingerprint Minutiae Matching Based on the Local and Global Structures," Proc. 15th Int'l Conf. Pattern Recognition, vol. 2, pp. 1038-1041, 2000.

[3] B. Bhanu and X.J. Tan, "Fingerprint Indexing Based on Novel Features of Minutiae Triplets," IEEE Trans. Pattern Analysis and Machine Intelligence, vol. 25, no. 5, pp. 616-622, May 2003.

[4] S. Gold and A. Rangarajan, "A Graduated Assignment Algorithm for Graph Matching," IEEE Trans. Pattern Analysis and Machine Intelligence, vol. 18, no. 4, pp. 377-388, Apr. 1996.

[5] Y.L. He, J. Tian, X.P. Luo, and T.H. Zhang, "Image Enhancement and Minutiae Matching in Fingerprint Verification," Pattern Recognition Letters, vol. 24/9-10, pp. 1349-1360, 2003.

[6] D. Maltoni, D. Maio, A.K. Jain, and S. Prabhakar, Handbook of Fingerprint Recognition. Springer-Verlag, 2003.

[7] A.K. Jain, S. Pankanti, and H. Lin, "A Multichannel Approach to Fingerprint Classification," IEEE Trans. Pattern Analysis and Machine Intelligence, vol. 21, no. 4, pp. 348-359, Apr. 1999.

[8] J.H. Chang and K.C. Fan, "A New Model for Fingerprint Classification by Ridge Distribution Sequences," Pattern Recognition, vol. 35, no. 6, pp. 1209-1223, June 2002.

[9] A.K. Jain, S. Prabhakar, L. Hong, and S. Pankanti, "FilterbankBased Fingerprint Matching," IEEE Trans. Image Processing, vol. 9, no. 5, pp. 846-859, May 2000.

[10] A. Teoh, D. Ngo, and O.T. Song, "An Efficient Fingerprint Verification System Using Integrated Wavelet and Fourier-Mellin Invariant Transform," Image and Vision Computing, vol. 22, no. 6, pp. 503-513, June 2004.

[11] V.A. Sujan and M.P. Mulqueen, "Fingerprint Identification Using Space Invariant Transforms," Pattern Recognition Letters, vol. 23, no. 5, pp. 609-619, Mar. 2002.

[12] C.J. Lee and S.D. Wang, "Fingerprint Feature Reduction by Principal Gabor Basis Function," Pattern Recognition, vol. 34, no. 11, pp. 2245-2248, Nov. 2001.

[13] A. Ross, A.K. Jain, and J. Reisman, "A Hybrid Fingerprint Matcher," Pattern Recognition, vol. 36, no. 7, pp. 1661-1673, July 2003.

[14] M. Tico and P. Kuosmanen, "Fingerprint Matching Using an Orientation-Based Minutia Descriptor," IEEE Trans. Pattern Analysis and Machine Intelligence, vol. 25, no. 8, pp. 1009-1014, Aug. 2003

[15] A.K. Jain, A. Ross, and S. Prabhakar, "Fingerprint Matching Using Minutiae and Texture Features," Proc. 2001 Int'l Conf. Image Processing, vol. 3, pp. 282-285, 2001.

[16] T.H. Zhang, J. Tian, Y.L. He, and X. Yang, "Fingerprint Alignment Using Similarity Histogram," Proc. Fourth Int'l Conf. Audio and Video-Based Biometric Person Authentication, pp. 854-861, 2003.

[17] X.P. Luo and J. Tian, "Knowledge Based Fingerprint Image Enhancement," Proc. 15th Int'l Conf. Pattern Recognition, vol. 4, pp. 783-786, 2000.

[18] Q.H. Xiao and H. Raafat, "Fingerprint Image Postprocessing: A Combined Statistical and Structural Approach," Pattern Recognition, vol. 24, no. 10, pp. 985-992, Oct. 1991.

[19] J.G Cheng and J. Tian, "Fingerprint Enhancement with Dyadic Scale-Space," Pattern Recognition Letters, vol. 25, pp. 1273-1284, 2004.

[20] D. Maio, D. Maltoni, R. Cappelli, J.L. Wayman, and A.K. Jain, "FVC2000: Fingerprint Verification Competition," IEEE Trans. Pattern Analysis and Machine Intelligence, vol. 24, no. 3, pp. 402-412, Mar. 2002.

[21] K.A. Toh et al., "Minutiae Data Synthesis for Fingerprint Identification Applications," Proc. 2001 Int'l Conf. Image Processing, vol. 2, pp. 262-265, 2001.

[22] R. Cappelli, D. Maio, and D. Maltoni, "Modelling Plastic Distortion in Fingerprint Images," Proc. Second Conf. Advances in Pattern Recognition, 2001.

[23] A.M. Bazen and S.H. Gerez, "Elastic Minutiae Matching by Means of Thin-Plate Spline Models," Proc. 16th Int'l Conf. Pattern Recognition, vol. 2, pp. 985-988, 2002.

[24] A. Senior and R. Bolle, "Improved Fingerprint Matching by Distortion Removal," IEICE Trans. Information and Systems, vol. E84-D, no. 7, pp. 825-831, July 2001.

[25] D. Maio, D. Maltoni, R. Cappelli, J.L. Wayman, and A.K. Jain, "FVC2002: Second Fingerprint Verification Competition," Proc. 16th Int'l Conf. Pattern Recognition, vol. 3, pp. 811-814, 2002. 
[26] D. Maio, D. Maltoni, R. Cappelli, J.L. Wayman, and A.K. Jain, "FVC2004: Third Fingerprint Verification Competition," Proc. First Int'l Conf. Biometric Authentication, pp. 1-7, July 2004.

[27] H. Chen, J. Tian, and X. Yang, "Fingerprint Matching with Registration Pattern Inspection," Proc. Fourth Int'l Conf. Audio and Video-Based Biometric Person Authentication, pp. 327-334, 2003.

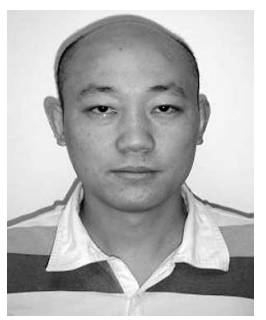

Yuliang He received the BS degree in computer and system science from Nankai University, China, in 1997 and the MS degree from the Institute of Automation, Chinese Academy of Sciences (CAS) in 2003. Now, he is a PhD candidate at CAS. His research interests include pattern recognition, machine learning, and information fusion and their applications in biometrics. He received the Dean Scholarship from CAS in 2005.

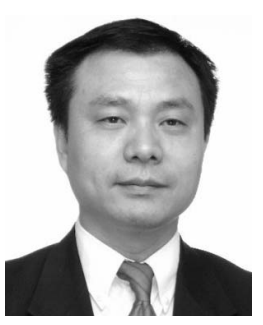

Jie Tian received the $\mathrm{PhD}$ degree (with honor) in artificial intelligence from the Institute of Automation, Chinese Academy of Sciences (CAS) in 1992. From 1994 to 1996, he was a postdoctoral fellow in the Medical Image Processing Group, University of Pennsylvania. Since 1997, he has been a professor in the Institute of Automation, CAS. His research interests are medical image process and analysis, pattern recognition, etc. $\mathrm{He}$ has published more than 50 papers in academic journals and international conferences. He received the National Science and Technology Advance Award in 2003 and 2004, respectively. He is a reviewerfor Mathematical Reviews. He is a senior member of the IEEE and the IEEE Computer Society.

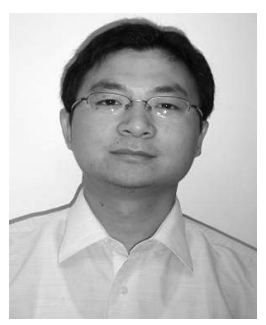

Liang Li received the BS degree from Northwestern Polytechnical University, China, in 2002. Now, he is a PhD candidate at the Chinese Academy of Sciences (CAS). His research interests include pattern recognition, machine learning, and image processing and their applications in biometrics.

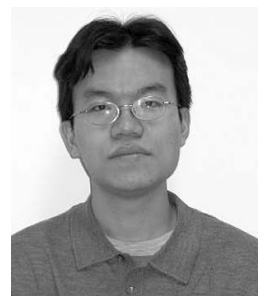

Hong Chen received the BS degree from Peking University, Beijing, China, in 2000 and the MS degree from the Institute of Automation, Chinese Academy of Sciences (CAS), in 2003. Now, he works at the Microsoft Research Advance Technology Center. His research interests include pattern recognition, computer vision, and image processing.

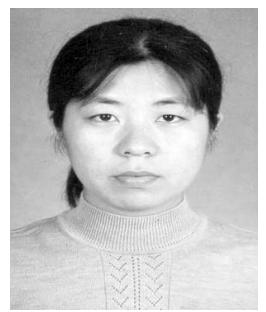

Xin Yang received the BS, MS, and $\mathrm{PhD}$ degrees in intelligent instruments from Tianjin University, China, in 1994, 1997, and 2000, respectively. From 2001 to 2003, she was a postdoctoral fellow at the Biometric Research Group, Key Laboratory of Complex Systems and Intelligence Science, Institute of Automation, Chinese Academy of Sciences (CAS). Since 2003, she has been an associate professor in the matics, pattern recognition, etc. same group. Her research interests are bioinfor-

$\triangleright$ For more information on this or any other computing topic, please visit our Digital Library at www.computer.org/publications/dlib. 